\title{
Homotopy Transfer Theorem for linearly compatible di-algebras
}

\author{
Yong Zhang
}

Received: 19 April 2012 / Accepted: 27 October 2012 / Published online: 30 November 2012

(C) Tbilisi Centre for Mathematical Sciences 2012

\begin{abstract}
This paper studies the operad of linearly compatible di-algebras, denoted by $A s^{2}$, which is a nonsymmetric operad encoding the algebras with two binary operations that satisfy individual and sum associativity conditions. We also prove that the operad $A s^{2}$ is exactly the Koszul dual operad of the operad ${ }^{2} A s$ encoding totally compatible di-algebras. We show that the operads $A s^{2}$ and ${ }^{2} A s$ are Koszul by rewriting method. We make explicit the Homotopy Transfer Theorem for $A s^{2}$-algebras.
\end{abstract}

Keywords Operad $\cdot$ Koszul $\cdot$ Di-algebra $\cdot$ Homotopy $\cdot$ Rewriting

\section{Introduction}

The motivation for the study of linearly compatible di-algebras (i.e., compatible associative products) can be found in $[1,10]$. In recent years, this algebraic structure was studied in [5,11-13] in connection with integrable systems, Cartan matrices of affine Dynkin diagrams, quiver representation and so on. For example, for a given linearly compatible di-algebra, one could construct a hierarchy of integrable systems of ODEs via Lenard-Magri Scheme [10]. If one of the multiplications was the usual product, then the integrable systems were the so-called top-like systems. These systems were exactly Hamiltonian $g l(N)$-modules with quadratic Hamiltonians [5]. In addition, A. V. Odesskii and V. V. Sokolov also discovered that the structure of linearly compatible di-algebra was more rigid than a compatible Lie algebra and therefore they had constructed the class of $M$-structures. These structures were in one-to-one correspondence with the linearly compatible di-algebras in semi-simple case over the complex field $\mathbb{C}$ in matrix. Moreover, their's results showed us that the classification of $M$-structures

Communicated by Hvedri Inassaridze.

Y. Zhang $(\bowtie)$

Department of Mathematics, Zhejiang University, Hangzhou 310027, China

e-mail: tangmeng@zju.edu.cn 
naturally led to the Cartan matrices of affine Dynkin diagram and the representations of such structures were described in terms of quiver representation.

On the other hand, the notion of associative algebra up to homotopy has been introduced by Jim Stasheff in [14] under the name $A_{\infty}$-algebra. It has the following important property: starting with a differential graded associative (dga) algebra $\left(A, d_{A}\right)$, if $\left(V, d_{V}\right)$ is a deformation retract of $\left(A, d_{A}\right)$, then $\left(V, d_{V}\right)$ is not a dga algebra in general, but an $A_{\infty}$-algebra. This is Kadeishvili's theorem [7], which is also called the Homotopy Transfer Theorem for associative algebras.

Our aim in this paper is to generalize the Homotopy Transfer Theorem for linearly compatible di-algebras. It means that we are going to determine the algebraic structure which is transferred to $V$. It consists in $n n$-ary operations, for any $n \geq 2$, which satisfy some relations analogous to the relations satisfied by the $n$-ary operations in an $A_{\infty}$-algebra. In [8] Loday and Vallette have given a generalization of the Homotopy Transfer Theorem for algebras over a Koszul operad, with explicit formulas. The key point is to make the Koszul dual cooperad explicit, and then to make also the differential map explicit in the cobar construction. First, we give a new proof of the theorem which says that the Koszul dual operad of linear compatible di-algebras is the operad of totally compatible di-algebras and that these two operads are Koszul. Second, we describe explicitly the composition in this operad ${ }^{2} A s$, so we obtain the relations satisfied by the generating operations.

Let $\mathbf{k}$ be a commutative unitary ring. The tensor product over $\mathbf{k}$ is denoted by $\otimes_{\mathbf{k}}$ or simply by $\otimes$ if it causes no confusion.

\section{Linearly compatible di-algebras}

We first recall the definition of linearly compatible di-algebra introduced in [15] by H. Strohmayer.

Remark 2.1 The operad of linearly compatible di-algebras is denoted by $A s^{\langle 2\rangle}$ in [18] and denoted by $A s^{2}$ in [15], in which Strohmayer has studied the compatible structures for various symmetric operads. From now on, in our paper, we choose the symbol $A s^{2}$ to denote the operad of linearly compatible di-algebras in nonsymmetric case.

Definition 2.1 A linearly compatible di-algebra is defined to be a k-module $V$ with two binary operations $*$ and $\bullet$ that are associative and satisfy the relation

$$
(x \bullet y) * z+(x * y) \bullet z=x \bullet(y * z)+x *(y \bullet z), \quad \forall x, y, z \in V .
$$

We give some examples of linearly compatible di-algebras as follows.

Example 2.1 (a) A matching di-algebra was introduced in [17] to be a k-module $V$ with two binary operations $*$ and $\bullet$ that are associative and satisfy the relation

$$
(x \bullet y) * z=x \bullet(y * z),(x * y) \bullet z=x *(y \bullet z), \quad \forall x, y, z \in V .
$$

Obviously any matching di-algebra is a linearly compatible di-algebra. It has a close relationship with a matched pair of two associative algebras and also gives 
rise to some important Lie type structures. Moreover, the operad of matching di-algebras is Koszul. In [18], the notion of matching di-algebra is denoted as $A s^{(2)}$-algebra.

(b) There are some examples of linearly compatible di-algebras from semi-homomorphisms. Recall that for a k-algebra $A$, a linear map $f: A \rightarrow A$ is called a left semi-homomorphism (resp. right semi-homomorphism) if $f$ satisfies

$$
f(x y)=x f(y)(\text { resp. } f(x y)=f(x) y), \quad \forall x, y \in A .
$$

A linear map $f: A \rightarrow A$ is called a semi-homomorphism if $f$ is both a left and a right semi-homomorphism. Therefore, for an associative $\mathbf{k}$-algebra $(A, \cdot)$, if $f$ is a left (resp. right) semi-homomorphism, then the triple $(A, *, \odot)$ with

$$
x * y:=x \cdot y, \quad x \odot y=f(x) \cdot y, \quad(\text { resp. } x \odot y=x \cdot f(y)), \quad \forall x, y \in A,
$$

is a linearly compatinle di-algebra. On the other hand, if $f, g$ are two semihomomorphisms, then the triple $(A, *, \odot)$ with

$$
\begin{aligned}
x * y & :=f(x) \cdot y(=f(x \cdot y)=x \cdot f(y)), \\
x \odot y & :=g(x) \cdot y(=g(x \cdot y)=x \cdot g(y)), \quad \forall x, y \in A,
\end{aligned}
$$

is a linearly compatible di-algebra, too.

(c) A duplicial algebra is a k-module $V$ determined by two binary operations $V \otimes V \rightarrow$ $V$ called left $(x, y) \mapsto x \prec y$ and $\operatorname{right}(x, y) \mapsto x \succ y$ respectively, satisfying the following three relations

$$
\begin{aligned}
& (x \prec y) \prec z=x \prec(y \prec z), \\
& (x \succ y) \prec z=x \succ(y \prec z),(x \succ y) \succ z=x \succ(y \succ z), \quad \forall x, y, z \in V .
\end{aligned}
$$

It is a direct consequence from above definition that the duplicial algebras can give rise to linearly compatible di-algebras. The author in [9] also showed that there was a good triple of operads (As, Dup, Mag).

The structure of free linearly compatible di-algebra and the generating function of the corresponding operad $A s^{2}$ were given in [3]. In [15], we know that the operad $A s^{2}$ of linearly compatible di-algebras is the black product of As and Lie $e_{2}$.

We observe that these three relations in 2.1 are equivalent to the associativity relation for the operation $(x, y) \mapsto \lambda(x \bullet y)+\mu(x * y)$ for any parameters $\lambda, \mu \in \mathbf{k}$.

\section{Totally compatible di-algebra and the operad $A s^{2}$}

In this section, we give the definition of totally compatible di-algebra and describe its associated nonsymmetric (ns) operad ${ }^{2} A s$. We also show that the operad ${ }^{2} A s$ is Koszul by the rewriting method. 
Definition 3.1 $[15,16]$ A totally compatible di-algebra is a k-module $V$ with two binary operations:

$$
*, \bullet: V \otimes V \rightarrow V
$$

satisfying the TCD axioms:

(a) $*$ and $\bullet$ are associative.

(b) $(x * y) \bullet z=x *(y \bullet z)=(x \bullet y) * z=x \bullet(y * z), \forall x, y, z \in V$.

Let ${ }^{2} A s$ denote the operad of totally compatible di-algebras as that in symmetric case in [15]. So we let ${ }^{2} A s$-algebra denote the totally compatible di-algebra. From the definition of totally compatible di-algebra, we see that the operad ${ }^{2} A s$ is nonsymmetric, set-theoretic, binary and quadratic. The structure of the ns operad ${ }^{2} A s$ can be derived from the following result.

Proposition 3.1 The vector space ${ }^{2} A s_{n}$ is $n$-dimensional, $n \geq 1$. Let $\mu_{i j}$ be the operation, given by

$$
\mu_{i j}\left(x_{1} \cdots x_{n}\right)=x_{1} * \cdots * x_{i+1} \bullet \cdots \bullet x_{n}
$$

with $i$ copies of $*$ and $j$ copies of $\bullet$. Then the composition in the operad ${ }^{2}$ As is given by

$$
\gamma\left(\mu_{i j} ; \mu_{i_{1} j_{1}}, \ldots, \mu_{i_{k} j_{k}}\right)=\mu_{i+i_{1}+\cdots+i_{k} j+j_{1}+\cdots+j_{k}} .
$$

In particular, we have

$$
\gamma\left(\mu_{10} ; \mu_{i j}, \mu_{k l}\right)=\mu_{i+j+1 j+l} ; \quad \gamma\left(\mu_{01} ; \mu_{i j}, \mu_{k l}\right)=\mu_{i+k j+l+1} .
$$

Proof In [16], we have proved that the triple $\left({ }^{2} A s(X):=\overline{\mathbf{k}<X>} \otimes \mathbf{k}<X>\right.$, *, $\left.\bullet\right)$ is the free totally compatible di-algebra on the set $X$, showing that the operad ${ }^{2} A s$ is $n$-dimensional in arity $n, n \geq 1$. Here $\mathbf{k}<X>$ denotes the free associative algebra over the set $X$, and $\overline{\mathbf{k}<X>}$ is its augmentation ideal.

Without loss of generality, for operations $\mu_{i j}$ and $\mu_{k l}$, given any element $x_{1} \cdots x_{n}$ in ${ }^{2} A s(X)$, we have

$$
\begin{aligned}
\gamma & \left(\mu_{i j} ; \mu_{i_{1} j_{1}}, \ldots, \mu_{i_{k} j_{k}}\right)\left(x_{1} \cdots x_{n}\right) \\
= & \mu_{i j}\left(\mu_{i_{1} j_{1}}\left(x_{1} \cdots x_{i_{1}+j_{1}+1}\right), \ldots, \mu_{i_{k} j_{k}}\left(x_{i_{1}+\cdots+i_{k-1}+j_{1}+\cdots+j_{k-1}+1} \cdots x_{n}\right)\right) \\
= & \mu_{i j}\left(x_{1} * \cdots * x_{i_{1}+1} \bullet \cdots \bullet x_{i_{1}+j_{1}+1},\right. \\
& \left.\quad \ldots, x_{i_{1}+\cdots+i_{k-1}+j_{1}+\cdots+j_{k-1}+1} * \cdots * x_{i_{1}+\cdots+i_{k-1}+j_{1}+\cdots+j_{k-1}+1+i_{k}} \bullet \cdots x_{n}\right) \\
= & x_{1} * \cdots * x_{i_{1}+\cdots+i_{k}+i+1} \bullet \cdots \bullet x_{n} \\
= & \mu_{i_{1}+\cdots+i_{k}+i j_{1}+\cdots+j_{k}+j}\left(x_{1} \cdots x_{n}\right)
\end{aligned}
$$

with $n-\left(i_{1}+\cdots+i_{k}+i+1\right)=j_{1}+\cdots+j_{k}+j$, implying the composition $\gamma$ of ${ }^{2} A s$. Then we get the ns operad ${ }^{2} A s=\left(\bigoplus_{n \geq 1}{ }^{2} A s_{n}, \gamma\right)$. 
In [15], Strohmayer has proved that the operad ${ }^{2} A s$ is Koszul by using the weight partition method. Here we give a different proof based on rewriting systems.

Theorem 3.1 The operad ${ }^{2}$ As is Koszul.

Proof Let $E$ be the generating space of binary operations with an ordered basis $\{*, \bullet\}$ such that $*>\bullet$. Let $\mu_{1}:=*$ and $\mu_{2}:=\bullet$.

Let $R$ be the space of relations, which is spanned by a set of relators written as in the following basis by the definition of totally compatible di-algebra in 3.1 :

Let $\circ_{1}:=((\cdot, \cdot), \cdot)$ and $\circ_{2}:=(\cdot,(\cdot, \cdot))$.

$$
\begin{aligned}
& \mu_{1} \circ_{1} \mu_{1}-\mu_{1} \circ_{2} \mu_{1}=0, \\
& \mu_{1} \circ_{1} \mu_{2}-\mu_{1} \circ_{2} \mu_{2}=0, \\
& \mu_{2} \circ_{1} \mu_{1}-\mu_{2} \circ_{2} \mu_{1}=0, \\
& \mu_{2} \circ_{1} \mu_{2}-\mu_{2} \circ_{2} \mu_{2}=0, \\
& \mu_{1} \circ_{2} \mu_{2}-\mu_{2} \circ_{2} \mu_{1}=0 .
\end{aligned}
$$

Let the monomials $\mu_{1} \circ_{1} \mu_{1}, \mu_{1} \circ_{1} \mu_{2}, \mu_{2} \circ_{1} \mu_{1}, \mu_{2} \circ_{1} \mu_{2}, \mu_{1} \circ_{2} \mu_{2}$ be the leading terms of relations $1-5$, respectively. Then the above choices provide rewriting rules of the form

$$
\begin{aligned}
& \mu_{i} \circ_{1} \mu_{j} \mapsto \mu_{i} \circ_{2} \mu_{j} \\
& \mu_{1} \circ_{2} \mu_{2} \mapsto \mu_{2} \circ_{2} \mu_{1}
\end{aligned}
$$

leading terms $\mapsto$ lower and non-leading terms

with $i, j \in\{1,2\}$, which give rise to the following critical monomials

$$
\left\{\begin{aligned}
\mu_{i} \circ_{1} \mu_{j} \circ_{1} \mu_{k}, i, j, k \in\{1,2\} & \text { if all the leading terms are in } 1-4 ; \\
\mu_{1} \circ_{2} \mu_{2} \circ_{1} \mu_{2}, \mu_{1} \circ_{1} \mu_{1} \circ_{2} \mu_{2}, & \mu_{2} \circ_{1} \mu_{1} \circ_{2} \mu_{2}, \mu_{1} \circ_{2} \mu_{2} \circ_{1} \mu_{1} \\
& \text { if there leading terms are in } 5 .
\end{aligned}\right.
$$

According to the rewriting method in chapter 8 in [8], it is enough to check that all the critical monomials in diagram 3 are confluent. We can see that relations 1-4 are of associative type. We know that the critical monomials of associative type are confluent, since their diamond is the following pentagon, see (Fig. 1).

In order to prove that the critical monomials of relation 5 are confluent, we take the first one in Eq. 5 as an example and the others can be proved in a similar way, see (Fig. 2).

Since all the critical monomials are confluent, the operad ${ }^{2}$ As is a Koszul operad.

Remark 3.1 (a) The rewriting method is due to E. Hoffbeck [6].

(b) The reader can find more details about rewriting method of ns operad in chapter 8 of [8] and [2].

Proposition 3.2 The operad As ${ }^{2}$ of linearly compatible di-algebras is the Koszul dual operad of the operad ${ }^{2}$ As. 


$$
\left(\mu_{i} \circ_{1} \mu_{j}\right) \circ_{1} \mu_{k}
$$

$$
\begin{array}{cc}
\swarrow & \searrow \\
\searrow & \mu_{i} \circ_{1}\left(\mu_{j} \circ_{2} \mu_{k}\right) \\
\left.\mu_{i} \circ_{2}\left(\mu_{j} \circ_{1} \circ_{k}\right) \mu_{k}\right) & \swarrow \\
\longrightarrow & \mu_{i} \circ_{2}\left(\mu_{j} \circ_{2} \mu_{k}\right)
\end{array}
$$

Fig. 1 The diamond for the associative type relations

$$
\mu_{1} \circ_{2} \mu_{2} \circ_{1} \mu_{2}
$$

$\left(\mu_{2} \circ_{2} \mu_{1}\right) \circ_{1} \mu_{2}$

$\mu_{1} \circ_{2}\left(\mu_{2} \circ_{1} \mu_{2}\right)$

$\downarrow$

$\downarrow$

$\mu_{2} \circ_{2}\left(\mu_{1} \circ_{1} \mu_{2}\right)$

$\mu_{1} \circ_{2}\left(\mu_{2} \circ_{2} \mu_{2}\right)$

$\downarrow$

$\downarrow$

$\mu_{2} \circ_{2}\left(\mu_{1} \circ_{2} \mu_{2}\right)$

$\mu_{2} \circ_{2}\left(\mu_{1} \circ_{2} \mu_{2}\right)$

$$
\mu_{2} \circ_{2}\left(\mu_{2} \circ_{2} \mu_{1}\right)
$$

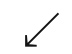

Fig. 2 The diamond for the first critical monomial of relation 5 
Remark 3.2 This result is a special case in Prop 1.14 in [15], if both the operads ${ }^{2} A s$ and $A s^{2}$ are seen as symmetric operads. Here we give a different proof when considered all the operads being nonsymmetric.

Proof Let $(\cdot 1, \cdot 2)_{1}$ denote the operation which sends $(x, y, z)$ to $((x \cdot 1 y) \cdot 2 z)$ and $(\cdot 1, \cdot 2)_{2}$ denote the operation which sends $(x, y, z)$ to $(x \cdot 1(y \cdot 2 z))$, with $\cdot 1, \cdot 2 \in\{*, \bullet\}$. The space $R$ of relations of ${ }^{2} A s$ is determined by the relators

$$
\left\{\begin{array}{c}
(*, *)_{2}-(*, *)_{1} \\
(\bullet, \bullet)_{2}-(\bullet, \bullet)_{1} \\
(\bullet, *)_{2}-(\bullet, *)_{1} \\
(*, \bullet)_{2}-(*, \bullet)_{1} \\
(\bullet, *)_{2}-(*, \bullet)_{1}
\end{array}\right\} .
$$

It is immediate to verify that its annihilator $R^{\perp}$, with respect to the given product in chapter 7 in [8], is the subspace determined by the following relators

$$
\left\{\begin{array}{l}
(*, *)_{2}-(*, *)_{1} \\
(\bullet, \bullet)_{2}-(\bullet, \bullet)_{1} \\
(\bullet, *)_{2}+(*, \bullet)_{2}-(\bullet, *)_{1}-(*, \bullet)_{2} .
\end{array}\right.
$$

These are precisely the expected relations in definition 2.1.

By [4] and [15], since the binary quadratic operad ${ }^{2} A s$ is a Koszul operad, it follows that its Koszul dual operad $A s^{2}$ is also Koszul.

\section{Homotopy Transfer Theorem for linearly compatible di-algebras}

In this section, we make explicit the notion of $A s^{2}$-algebra up to homotopy i.e., $A s_{\infty}^{2}$ algebra by describing the $\mathrm{dg}$ operad $A s_{\infty}^{2}$.

Since the operad $A s^{2}$ is Koszul, the $\operatorname{dg}$ operad $A s_{\infty}^{2}$ is given by $A s_{\infty}^{2}:=\Omega\left(\left(A s^{2}\right)^{\mathrm{i}}\right)$. So we need to describe $\left(A s^{2}\right) i=\left({ }^{2} A s\right)^{*}$, which is the co-operad linearly dual to ${ }^{2} A s$. By proposition 3.1, we know that the space $\left({ }^{2} A s\right)_{n}^{*}$ is $n$-dimensional. In order to describe the differential of the cobar construction $A s_{\infty}^{2}$, we need to introduce the following definition and lemma.

Definition 4.1 (chapter 6 in [8]) For any co-operad $(\mathcal{C}, \Delta, \eta)$ with counit $\eta: \mathcal{C} \rightarrow I$, we consider the projection of the decomposition map to the infinitesimal part of the composite product $\mathcal{C} \circ \mathcal{C}$. This map is called the infinitesimal decomposition map of $\mathcal{C}$ and is defined by the following composite

$$
\Delta_{(1)}:=\mathcal{C} \stackrel{\Delta}{\rightarrow} \mathcal{C} \circ \mathcal{C} \stackrel{\operatorname{Id}_{\mathcal{C}}{ }^{\prime} \operatorname{Id}_{\mathcal{C}}}{\longrightarrow} \mathcal{C} \circ(\mathcal{C} ; \mathcal{C}) \stackrel{\operatorname{Id}_{\mathcal{C}} \circ\left(\eta ; \operatorname{Id}_{\mathcal{C}}\right)}{\longrightarrow} \mathcal{C} \circ(\mathrm{I} ; \mathcal{C})=\mathcal{C} \circ{ }_{(1)} \mathcal{C}
$$

where the notation $\operatorname{Id}_{\mathcal{C}} \circ^{\prime} \operatorname{Id}_{\mathcal{C}}$ is the infinitesimal composite of $\operatorname{Id}_{\mathcal{C}}$ and $\operatorname{Id}_{\mathcal{C}}$ and the notation $\mathcal{C}{ }_{{ }_{(1)}} \mathcal{C}$ denotes the infinitesimal composite of $\mathcal{C}$ and $\mathcal{C}$.

Remark $4.1 \Delta_{(1)}$ is also called the linear part of the co-composition $\Delta$. 
Lemma 4.1 (chapter 6 in [8]) For a given co-operad $(\mathcal{C}, \Delta, \eta)$, the dg cobar construction of the co-operad $\mathcal{C}$ is given by

$$
\Omega(\mathcal{C}):=\left(\mathcal{T}\left(s^{-1} \overline{\mathcal{C}}\right), d\right)
$$

with d induced by $\Delta_{(1)}$ as follows

$$
\begin{aligned}
& d: \mathbf{k} s^{-1} \otimes \overline{\mathcal{C}} \stackrel{\Delta_{s} \otimes \Delta_{(1)}}{\longrightarrow}\left(\mathbf{k} s^{-1} \otimes \mathbf{k} s^{-1}\right) \otimes\left(\overline{\mathcal{C}}{ }_{(1)} \overline{\mathcal{C}}\right) \\
& \quad \stackrel{I d \otimes \tau \otimes I d}{\longrightarrow}\left(\mathbf{k} s^{-1} \otimes \overline{\mathcal{C}}\right){ }_{(1)}\left(\mathbf{k} s^{-1} \otimes \overline{\mathcal{C}}\right) \cong \mathcal{T}\left(s^{-1} \overline{\mathcal{C}}\right)^{(2)} \longmapsto \mathcal{T}\left(s^{-1} \overline{\mathcal{C}}\right),
\end{aligned}
$$

where $s$ is the decoration, modifying the degree of the objects in $\overline{\mathcal{C}}$.

From lemma 4.1, it is sufficient to make explicit the infinitesimal part $\Delta_{(1)}$ in the operad $\left({ }^{2} A s\right)^{*}$ to get the differential map $\partial$ of cobar construction $A s_{\infty}^{2}=\mathcal{T}\left(\left(A s^{2}\right)^{i}\right)$, without decoration $s$.

Theorem 4.1 The linear part of the co-composition $\Delta$ in $\left({ }^{2} A s\right)^{*}$ is given by

$$
\Delta_{(1)}\left(\mu_{c d}^{c}\right)=\sum_{\substack{c=i+a \\ d=j+b}} \mu_{i j}^{c}(\underbrace{i d, \ldots, i d}_{p \text { copies }}, \mu_{a b}^{c}, \underbrace{i d, \ldots, i d}_{r \text { copies }})
$$

excluding $(a, b)=(i, j)=(0,0)$ with $\mu_{i j} \in{ }^{2} A s_{n}, i+j=n-1$.

Proof By the property of linearly dual basis, it is a straightforward computation. Since

$$
\gamma(\mu_{i j} ; \underbrace{i d, \ldots, i d}_{p \text { copies }}, \mu_{a, b}, \underbrace{i d, \ldots, i d}_{r \text { copies }})=\mu_{i+a j+b},
$$

then

$$
\Delta_{(1)}\left(\mu_{c d}^{c}\right)=\sum_{c=i+a, d=j+b} \mu_{i j}^{c}(\underbrace{i d, \ldots, i d}_{p \text { copies }}, \mu_{a b}^{c}, \underbrace{i d, \ldots, i d}_{r \text { copies }}) .
$$

Let $m_{i j}:=\mu_{i j}^{c}$ be the generator of the cobar construction $A s_{\infty}^{2}$. Then we get the following result.

Theorem 4.2 The operad As $s_{\infty}^{2}$ is generated by the operations $m_{i j}$, with $\left|m_{i j}\right|=n-2$ for $i+j=n-1, i, j \geq 0$, which satisfy the following formula:

$$
\partial\left(m_{i j}\right)=\sum_{\begin{array}{c}
a+c=i, b+d=j \\
q=c+d+1, p+q+r=i+j+1
\end{array}}(-1)^{p+q r} m_{a b}(\underbrace{i d, \ldots, i d}_{p \text { copies }}, m_{c d}, \underbrace{i d, \ldots, i d}_{r \text { copies }}) .
$$

Proof From the definition of $A s_{\infty}^{2}$ and Theorem 4.1, it follows that $A s_{\infty}^{2}$ is generated by the operations $m_{i j}$. 
By definition of the cobar construction in lemma 4.1, the boundary map on $\Omega\left(\left(A s^{2}\right)^{\mathrm{i}}\right)$ is induced by the co-operad structure of $\left(A s^{(2)}\right)^{\mathrm{i}}$, and more precisely by $\Delta_{(1)}$ of linear dual co-operad $\left({ }^{2} A s\right)^{*}$ given by Theorem 4.1 as:

$$
\Delta_{(1)}\left(\mu_{c d}^{c}\right)=\sum_{\begin{array}{c}
c=i+a \\
d=j+b
\end{array}} \mu_{i j}^{c}(\underbrace{i d, \ldots, i d}_{p \text { copies }}, \mu_{a b}^{c}, \underbrace{i d, \ldots, i d}_{r \text { copies }})
$$

excluding $(a, b)=(i, j)=(0,0)$ with $\mu_{i j} \in{ }^{2} A s_{n}, i+j=n-1$. By the construction of the differential given in lemma 4.1, we have

$$
\partial\left(m_{i j}\right)=\sum_{\begin{array}{c}
a+c=i, b+d=j \\
q=c+d+1, p+q+r=i+j+1
\end{array}}(-1)^{p+q r} m_{a b}(\underbrace{i d, \cdots, i d}_{p \text { copies }}, m_{c d}, \underbrace{i d, \cdots, i d}_{r \text { copies }}) .
$$

The signs are obtained by comparison with the $\operatorname{dg}$ operad $A_{\infty}$.

Now, we introduce the Homotopy Transfer Theorem for Koszul operads.

Theorem 4.3 ([8]) (Homotopy Transfer Theorem). Let $\mathcal{P}$ be a Koszul operad and let $\left(V ; d_{V}\right)$ be a homotopy retract of $\left(W ; d_{W}\right)$ :

$$
\begin{aligned}
& h \bigcirc_{1}\left(W, d_{W}\right) \frac{p}{i} \underset{\longrightarrow}{\longrightarrow}\left(V, d_{V}\right) \\
& I d_{W}-i p=d_{W} h+h d_{W},
\end{aligned}
$$

where the chain map $i$ is a quasi-isomorphism. Any $\mathcal{P}_{\infty}$-algebra structure on $W$ can be transferred into a $\mathcal{P}_{\infty}$-algebra structure on $V$ such that $i$ extends to an $\infty$-quasiisomorphism.

From the above results, we get the Homotopy Transfer Theorem for the operad $A s^{2}$.

Theorem 4.4 Let

$$
\begin{gathered}
h C_{1}\left(A, d_{A}\right)_{i} \ldots\left(V, d_{V}\right) \\
i=\text { quasi-isomorphism } I d_{A}-i p=d_{A} h+h d_{A},
\end{gathered}
$$

be a homotopy retract. If $\left(A, d_{A}\right)$ is a dg $A s^{2}$-algebra, then $\left(V, d_{V}\right)$ inherits an $A s^{2} \infty^{-}$ algebra structure $\left\{m_{i j}\right\}_{i+j=n-1}$ with $n \geq 2$, which extend functorially the binary operations of $A$.

Proof The conclusion is a direct consequence of the Homotopy Transfer Theorem following from that the operad $A s^{2}$ is Koszul.

Acknowledgments I am greatly indebted to my supervisors Professor Jean-Louis Loday (CNRS and Strasbourg University) and Professor Fang Li (Zhejiang University) for their constant support. Part of this work was achieved in Strasbourg, France, during a visit sponsored by the Centre National de la Recherche Scientifique. 


\section{References}

1. Cariñena, J.F., Grabowski, J., Marmo, G.: Quantum bi-Hamiltonian systems. J. of Modern. Phys. A 15(30), 4797-4810 (2000)

2. Dotsenko, V., Khoroshkin, S.: Gröbner bases for operads. Duke Math. J. 153(2), 363-396 (2010)

3. Dotsenko, V.: Compatible associative products and trees. Algebra Number Theory 3(5), 567-586 (2009)

4. Ginzburg, V., Kapranov, M.: Koszul duality for operads. Duke Math. J. 76(1), 203-272 (1994)

5. Golubchik, I.Z., Sokolov, V.V.: Compatible Lie brackets and the Yang-Baxter equation. Theor. Math. Phys. 146, 159-169 (2006)

6. Hoffbeck, E.: A Poincaré-Birkhoff-Witt criterion for Koszul operads. Manuscripta Math. 131, 87-110 (2010)

7. Kadeishvili, T. V.: The algebraic structure in the homology of an A(1)-algebra, Soobshch. Akad. Nauk Gruzin. SSR 108 (1982), no. 2, 249-252 (1983)

8. Loday, J.-L., Vallette, B.: Algebraic operads, Grundlehren Math. Wiss. 346, Springer, Heidelberg, (2012)

9. Loday, J.-L.: Generalized bialgebras and triples of operads, Astérisque, no. 320, x+116p (2008)

10. Magri, F.: A simple model of the integrable Hamiltonian equation. J. Math. Phys. 19:1156C1162 (1978)

11. Odesskii, A.V., Sokolov, V.V.: Algebraic structures connected with pairs of compatible associative algebras. Int. Math. Res. Notices (2006). doi:10.1155/IMRN/2006/43734

12. Odesskii, A.V., Sokolov, V.V.: Integrable matrix equations related to pairs of compatible associative algebras. J. Phys. A: Math. Gen. 39, 12447-12456 (2006)

13. Odesskii, A.V., Sokolov, V.V.: Pairs of compatible associative algebras, classical Yang-Baxter equation and quiver representations. Commun. Math. Phys. 278, 83-99 (2008)

14. Stasheff, J.D.: Homotopy associativity of H-spaces. I, II.. Trans. Amer. Math. Soc. 108:275-292, 293-312 (1963)

15. Strohmayer, H.: Operads of compatible structures and weighted partitions. J. Pure Appl. Algebra 212, 2522-2534 (2008)

16. Zhang, Y., Bai C.M., Guo, L.: Totally compatible associative and lie dialgebras, tridendriform algebras and postLie algebras. Preprint (2011)

17. Zhang, Y., Bai, C.M., Guo, L.: The category and operad of matching dialgebras. Appl. Categor. Struct. (2012). doi:10.1007/s10485-012-9290-7

18. Zinbiel, G.W.: Encyclopedia of types of algebras 2010. In: Proceedings of the International Conference in Nankai Series in Pure, Applied Mathematics and Theoretical Physics, vol. 9 (World Scientific, Singapore), 219-298 (2012) 